\title{
On a problem for the loaded degenerating mixed type equation involving integral-differential operators
}

\author{
B. I. Islomov ${ }^{1}$, O. Kh. Abdullayev ${ }^{1}$, N. K. Ochilova ${ }^{2}$ \\ ${ }^{1}$ National University of Uzbekistan, Universitetskaya-4, VUZgorodog, Tashkent, 100174, Uzbekistan \\ ${ }^{2}$ Tashkent financial institute, st. Amir Timur. 57, Tashkent, 100000, Uzbekistan \\ islomovbozor@yandex.ru, obidjon.mth@gmail.com,nargiz.ochilova@gmail.com
}

PACS 2010 MSC-35M10

DOI 10.17586/2220-8054-2017-8-3-323-333

\begin{abstract}
This work aims to study the existence and uniqueness of a solution for a problem of the loaded degenerating mixed type equation. We consider the loaded parabolic-hyperbolic equation involving the Caputo fractional derivative and Riemann-Liouville integrals. The uniqueness of solution is proved by using the method of integral energy applying an extremum principle. Based on the statement of equivalence between "The existence and uniqueness of solution" and "Solvability of the respectively Fredholm type integral equations", the existence of a solution was proved.
\end{abstract}

Keywords: loaded degenerating equation, parabolic-hyperbolic type, integral operators, Caputo fractional derivative, existence and uniqueness of solution, integral equations.

Received: 18 May 2017

Revised: 30 May 2017

\section{Introduction}

The first, most general definitions of loaded equations and various loaded equations were classified in detail by A. M. Nakhushev [1].

Definition 1. An equation $A u(x)=f(x)$ is called a loaded equation in $\mathrm{n}$ dimensional Euclidean domain $\Omega$ if the operator $A$ depends of the restriction of the unknown function to a closed subset of $\bar{\Omega}$, of measure strictly less than $n$.

To that end, some local and non-local problems were investigated for the loaded mixed type equation involving fractional order integral-differential operators (see [2,3]). In the works [4-7] we can see significant development in the field of fractional differential equations in recent years. Various phenomena in physics, like diffusion in a disordered or fractal medium, or in image analysis, or in risk management have been modeled by means of fractional partial differential equations. In general, there exists no method that yields an exact solution for these equations. Indeed, we can find numerous applications in viscoelasticity, neurons, electrochemistry, control, porous media, electromagnetism, etc., (for details, see [8-11] ). See Ref. [12] for deterministic fractional models in bioengineering and nanotechnology. Fractional calculus is widely applied to the investigation of partial differential equations of mixed type and hyperbolic type with degenerations (see [7,13,14] ). In a series of papers (see [15-17]), the authors considered some classes of boundary value problems for mixed type non degenerating and degenerating differential equations involving Caputo and Riemann-Liouville fractional derivatives of order $0<\alpha \leq 1$.

\section{Preliminaries}

\subsection{Riemann-Liouville and Caputo integral-differential operators}

Definition 2. Let $f(x)$ be an absolutely continuous function over $(a, b)$. Then the left and right Riemann-Liouville fractional integrals order $\alpha\left(\alpha \in \mathbb{R}^{+}\right)$(respectively) are (see [4], p. 69)

$$
\begin{aligned}
& \left(I_{a+}^{\alpha} f\right) x=\frac{1}{\Gamma(\alpha)} \int_{a}^{x} f(t)(x-t)^{\alpha-1} d t, \quad x>a \\
& \left(I_{-b}^{\alpha} f\right) x=\frac{1}{\Gamma(\alpha)} \int_{x}^{b} f(t)(t-x)^{\alpha-1} d t, \quad x<b .
\end{aligned}
$$


Definition 3. The Riemann-Liouville fractional derivatives $D_{a x}^{\alpha} f$ and $D_{x b}^{\alpha} f$ of order $\alpha\left(\alpha \in \mathbb{R}^{+}\right)$, are defined by (see [4], p. 70):

$$
\begin{gathered}
\left(D_{a x}^{\alpha} f\right) x=\frac{1}{\Gamma(n-\alpha)}\left(\frac{d}{d x}\right)^{n} \int_{a}^{x} \frac{f(t)}{(x-t)^{\alpha-n+1}} d t, n=[\alpha]+1, \quad x>a ; \\
\left(D_{x b}^{\alpha} f\right) x=\frac{1}{\Gamma(n-\alpha)}\left(-\frac{d}{d x}\right)^{n} \int_{x}^{b} \frac{f(t)}{(t-x)^{\alpha-n+1}} d t, \quad n=[\alpha]+1, \quad x<b ;
\end{gathered}
$$

respectively, where $[\alpha]$ is the integer part of $\alpha$. In particular, for $\alpha=N \cup\{0\}$ we have $\left(D_{a x}^{0} f\right) x=f(x)$, $\left(D_{x b}^{0} f\right) x=f(x),\left(D_{a x}^{n} f\right) x=f^{(n)}(x) ;\left(D_{x b}^{n} f\right) x=(-1)^{n} f^{(n)}(x), n \in \mathbb{N}$, where $f^{(n)}(x)$ is the usual derivative of $f(x)$ of order $n$.

Definition 4. Caputo fractional derivatives ${ }_{C} D_{a x}^{\alpha} f$ and ${ }_{C} D_{x b}^{\alpha} f$ of order $\alpha>0(\alpha \notin \mathbb{N} \cup\{0\})$ are defined by (see [4], p. 92):

$$
\begin{aligned}
& \left({ }_{C} D_{a x}^{\alpha} f\right) x=\frac{1}{\Gamma(n-\alpha)} \int_{a}^{x} \frac{f^{(n)}(t)}{(x-t)^{\alpha-n+1}} d t, \quad n=[\alpha]+1, \quad x>a \\
& \left({ }_{C} D_{x b}^{\alpha} f\right) x=\frac{(-1)^{n}}{\Gamma(n-\alpha)} \int_{x}^{b} \frac{f^{(n)}(t)}{(t-x)^{\alpha-n+1}} d t, \quad n=[\alpha]+1, \quad x<b ;
\end{aligned}
$$

respectively. From (3)-(6), as a conclusion we will have: $k-1<\alpha \leq k, k \in \mathbb{N}$; consequently, while for $\alpha \in \mathbb{N} \cup\{0\}$ we have

$$
\begin{gathered}
\left({ }_{C} D_{a x}^{0} f\right) x=f(x), \quad\left({ }_{C} D_{x b}^{0} f\right) x=f(x), \\
\left(_{C} D_{a x}^{n} f\right) x=f^{(n)}(x), \quad\left({ }_{C} D_{x b}^{n} f\right) x=(-1)^{n} f^{(n)}(x), n \in \mathbb{N} .
\end{gathered}
$$

\subsection{Gauss hypergeometric function}

Gauss hypergeometric function $F(a, b, c, z)$ is defined in the unit desk as the sum of the hypergeometric series (see [4], p. 27):

$$
F(a, b, c, z)=\sum_{k=0}^{\infty} \frac{(a)_{k}(b)_{k}}{(c)_{k}} \frac{z^{k}}{k !}
$$

where $|z|<1, a, b \in \mathbb{C}, c \in \mathbb{C} \backslash Z_{0}^{-}$and $(a)_{0}=1, \quad(a)_{n}=a(a+1) \ldots(a+n-1)=\frac{\Gamma(a)}{\Gamma(a+n)},(n=1,2, \ldots)$.

One such analytic continuation is given by Euler integral representation:

$$
F(a, b, c ; z)=\frac{\Gamma(c)}{\Gamma(b) \Gamma(c-b)} \int_{0}^{1} x^{b-1}(1-x)^{c-b-1}(1-z x)^{-a} d x,
$$

$0<\operatorname{Re} b<\operatorname{Re} c,|\arg (1-z)|<\pi$. The Gauss hypergeometric function $F(a, b, c, z)$ allows the following estimation:

$$
F(a, b, c ; z) \leq \begin{cases}c_{1}, & \text { if } c-a-b>0, \quad 0 \leq z \leq 1 \\ c_{2}(1-z)^{c-a-b}, & \text { if } c-a-b<0, \quad 0<z<1 \\ c_{3}(1+|\ln (1-z)|), & \text { if } c-a-b=0 .\end{cases}
$$

\subsection{Wright type functions}

The elementary definition of the Wright type function at $\alpha>\beta, \alpha>0$ and for all $z \in \mathbb{C}$, is [18]

$$
e_{\alpha, \beta}^{\mu, \delta}(z)=\sum_{k=0}^{\infty} \frac{z^{n}}{\Gamma(\alpha n+\mu) \Gamma(\delta-\beta n)} .
$$

If $\alpha=\mu=1$, then from (10) we have:

$$
e_{1, \beta}^{1, \delta}(z)=\sum_{k=0}^{\infty} \frac{z^{n}}{n ! \Gamma(\delta-\beta n)}
$$




\section{Problem formulation and main functional relation}

We consider equation:

$$
0= \begin{cases}u_{x x}-{ }_{C} D_{o y}^{\alpha} u+\sum_{k=1}^{n} p_{k} I_{0 x}^{\beta_{k}} u(x, 0), & \text { at } y>0 \\ (-y)^{m} u_{x x}-x^{m} u_{y y}+\sum_{k=1}^{n} q_{k} I_{0 \eta}^{\gamma_{k}} u\left(\eta^{\frac{1}{m+2}}, 0\right), & \text { at } y<0\end{cases}
$$

with operators (see (1) and (5)):

$$
{ }_{C} D_{o y}^{\alpha} u=\frac{1}{\Gamma(1-\alpha)} \int_{0}^{y}(y-t)^{-\alpha} u_{t}(x, t) d t, \quad\left(I_{a x}^{\beta} u\right)(x, 0)=\frac{1}{\Gamma(\beta)} \int_{a}^{x}(x-t)^{\beta-1} u(t, 0) d t,
$$

where $0<\alpha, \beta_{k}, \gamma_{k}<1, \xi=x^{\frac{m+2}{2}}-(-y)^{\frac{m+2}{2}}, m, p_{k}, q_{k}=$ const, $m>0$.

We set the $\Omega$ domain, bounded with segments: $A_{1} A_{2}=\{(x, y): x=1,0<y<h\}, B_{1} B_{2}=\{(x, y)$ : $x=0,0<y<h\}, B_{2} A_{2}=\{(x, y): y=h, 0<x<1\}$ at the $y>0$, and by the characteristics: $A_{1} C: x^{\frac{m+2}{2}}+(-y)^{\frac{m+2}{2}}=1 ; B_{1} C: x^{\frac{m+2}{2}}-(-y)^{\frac{m+2}{2}}=0$ of equation (12) at $y<0$, where $A_{1}(1 ; 0), A_{2}(1 ; h)$, $B_{1}(0 ; 0), B_{2}(0 ; h), C\left(2^{-\frac{2}{m+2}} ;-2^{-\frac{2}{m+2}}\right)$. Introduce designations: $\Omega^{+}=\Omega \cap(y>0), \Omega^{-}=\Omega \cap(y<0)$.

For equation (12), we consider the following problem: find a solution $u(x, y)$ of equation (12) from the following class of functions:

$$
W=\left\{u(x, y): u(x, y) \in C(\bar{\Omega}) \cap C^{2}\left(\Omega^{-}\right), \quad u_{x x} \in C\left(\Omega^{+}\right), \quad{ }_{C} D_{o y}^{\alpha} u \in C\left(\Omega^{+}\right)\right\}
$$

satisfies boundary conditions:

and gluing conditions:

$$
\begin{gathered}
\left.u(x, y)\right|_{A_{1} A_{2}}=\varphi(y), 0 \leq y \leq h, \\
\left.u(x, y)\right|_{B_{1} B_{2}}=\psi(y), 0 \leq y \leq h, \\
\left.u(x, y)\right|_{B_{1} C}=h(x), \quad 0 \leq x \leq 2^{-\frac{2}{m+2}} .
\end{gathered}
$$

$$
\lim _{y \rightarrow+0} y^{1-\alpha} u_{y}(x, y)=\lambda u_{y}(x,-0), \quad(x, 0) \in A_{1} B_{1},
$$

where $\varphi(y), \psi(y), h(x)$ are given functions and $\lambda=$ const $\left(\lambda \in \mathbb{R}^{+}\right)$. In order to solve the above problem we need the following Riemann function.

Its known [19] that the Riemann function for equation (12) at $y<0$ on the characteristics coordinate $\xi=x^{\frac{m+2}{2}}-(-y)^{\frac{m+2}{2}}$ and $\eta=x^{\frac{m+2}{2}}+(-y)^{\frac{m+2}{2}}$ defined with the Gauss hypergeometric function

$$
R\left(\xi_{0}, \eta_{0} ; \xi, \eta\right)=\frac{\left(\eta^{2}-\xi^{2}\right)^{2 \delta}}{\left(\eta^{2}-\xi_{0}^{2}\right)^{\delta}\left(\eta_{0}^{2}-\xi^{2}\right)^{\delta}} F\left(\delta, \delta, 1 ; \frac{\left(\xi_{0}^{2}-\xi^{2}\right)\left(\eta^{2}-\eta_{0}^{2}\right)}{\left(\eta^{2}-\xi_{0}^{2}\right)\left(\eta_{0}^{2}-\xi^{2}\right)}\right),
$$

where $\delta=\frac{m}{2(m+2)}$. In fact, that a solution of the Cauchy problem for equation (12) in the domain $\Omega^{-}$with initial dates

$$
\begin{gathered}
u(x, 0)=\tau(x), \quad 0 \leq x \leq 1 \\
u_{y}(x,-0)=\nu^{-}(x), \quad 0<x<1,
\end{gathered}
$$

will be given by formula:

$$
\begin{gathered}
u(x, y)=k_{1} \int_{\xi}^{\eta}\left(t^{2}-\xi^{2}\right)^{-\delta}\left(\eta^{2}-t^{2}\right)^{-\delta} \nu^{-}\left(t^{1-2 \delta}\right) d t- \\
k_{2} \int_{\xi}^{\eta}\left(\eta^{2}-\xi^{2}\right)^{1-2 \delta}\left(t^{2}-\xi^{2}\right)^{\delta-1}\left(\eta^{2}-t^{2}\right)^{\delta-1} t \tau^{-}\left(t^{1-2 \delta}\right) d t+ \\
\sum_{k=1}^{n} \int_{\xi}^{\eta} q_{k} I_{0 t^{2}}^{\gamma_{k}} \tau\left(t^{1-2 \delta}\right) d t \int_{t}^{\eta} \frac{\left(\eta^{2}-\xi^{2}\right)^{2 \delta}}{\left(\eta^{2}-t^{2}\right)^{\delta}\left(z^{2}-\xi^{2}\right)^{\delta}} F\left(\delta, \delta, 1 ; \frac{\left(t^{2}-\xi^{2}\right)\left(\eta^{2}-z^{2}\right)}{\left(\eta^{2}-t^{2}\right)\left(z^{2}-\xi^{2}\right)}\right) d z
\end{gathered}
$$


where

$$
k_{1}=\frac{\Gamma(2 \delta)}{\Gamma^{2}(\delta)}, \quad k_{2}=\frac{\Gamma(1-2 \delta)}{2^{1-2 \delta} \Gamma^{2}(1-\delta)}\left(\frac{m+2}{2}\right)^{-2 \delta} .
$$

Let us find the relation between $\tau^{-}(x)$ and $\nu^{-}(x)$ transferred from the hyperbolic part $\Omega^{-}$to the line $y=0$. By using condition (16) and taking (13) into account, from (20) we obtain:

$$
\begin{aligned}
\tilde{\nu}^{-}\left(\eta^{1-2 \delta}\right)= & \frac{k_{2} \Gamma(1-\delta)}{k_{1} \Gamma(\delta)} \eta D_{0}^{1-2 \delta} \tilde{\tau}\left(\eta^{1-2 \delta}\right)-\frac{2 \Gamma(1-\delta)}{k_{1}} \eta^{2 \delta} D_{0}^{1-\delta} \eta^{2} \int_{0}^{\eta} \sum_{k=1}^{n} \frac{q_{k}}{\Gamma\left(\gamma_{k}\right)} d t \int_{0}^{t^{2}}\left(t^{2}-s\right)^{\gamma_{k}-1} \tilde{\tau}\left(s^{1 / 2-\delta}\right) d s \times \\
& \int_{t}^{\eta} \frac{\left(\eta^{2}\right)^{2 \delta}}{\left(\eta^{2}-t^{2}\right)^{\delta}\left(z^{2}\right)^{\delta}} F\left(\delta, \delta, 1 ; \frac{t^{2}\left(\eta^{2}-z^{2}\right)}{z^{2}\left(\eta^{2}-t^{2}\right)}\right) d z+\frac{2 \Gamma(1-\delta)}{k_{1}} \eta^{2 \delta} D_{0}^{1-\delta} \eta^{2} h\left[\left(\frac{\eta}{4}\right)^{\frac{2}{m+2}}\right] .
\end{aligned}
$$

Moreover, replacing $\eta^{2} \sim \eta_{1}, t^{2} \sim t, z^{2} \sim z$ we have:

$$
\begin{aligned}
\tilde{\nu}^{-}(\eta)= & \frac{k_{2} \Gamma(1-\delta)}{k_{1} \Gamma(\delta)} \eta_{1}{ }^{1 / 2} D_{0}^{1-2 \delta} \tilde{\tau}(\eta)-\frac{\Gamma(1-\delta)}{2 k_{1}} \eta_{1}{ }^{\delta} D_{0}^{1-\delta} \int_{1} \int_{0}^{\eta_{1}} \sum_{k=1}^{n} \frac{q_{k} t^{1 / 2}}{\Gamma\left(\gamma_{k}\right)} d t \int_{0}^{t}(t-s)^{\gamma_{k}-1} \tilde{\tau}(s) d s \times \\
& \int_{t}^{\eta_{1}} \frac{\eta_{1}{ }^{2 \delta}}{\left(\eta_{1}-t\right)^{\delta} z^{\delta+1 / 2}} F\left(\delta, \delta, 1 ; \frac{t\left(\eta_{1}-z\right)}{z\left(\eta_{1}-t\right)}\right) d z+\frac{2 \Gamma(1-\delta)}{k_{1}} \eta_{1}{ }^{\delta} D_{0}^{1-\delta} h\left[\left(\frac{\eta_{1}}{4}\right)^{\frac{1}{m+2}}\right]
\end{aligned}
$$

were

$$
\begin{gathered}
\tilde{\nu}^{-}(\eta)=\nu^{-}\left(\eta^{1-2 \delta}\right)=\nu^{-}\left(\eta_{1}{ }^{1 / 2-\delta}\right), \\
\tilde{\tau}(\eta)=\tau\left(\eta^{1-2 \delta}\right)=\tau\left(\eta_{1}{ }^{1 / 2-\delta}\right) .
\end{gathered}
$$

Considering designations (19) and $\lim _{y \rightarrow+0} y^{1-\alpha} u_{y}(x, y)=\nu^{+}(x), 0<x<1$ from gluing condition (17), we have

$$
\nu^{+}(x)=\lambda \nu^{-}(x)
$$

For further consideration, from Eq. (12) at $y \rightarrow+0$ considering (13), (22) and

$$
\lim _{y \rightarrow 0} D_{0 y}^{\alpha-1} f(y)=\Gamma(\alpha) \lim _{y \rightarrow 0} y^{1-\alpha} f(y)
$$

we obtain:

$$
\tau^{\prime \prime}(x)-\Gamma(\alpha) \nu^{+}(x)+\sum_{k=1}^{n} p_{k} I_{0 x}^{-\beta_{k}} \tau(x)=0 .
$$

\section{Uniqueness of the solution}

Theorem 1. If satisfy conditions $\lambda>0$ and

$$
0<\alpha, \beta_{k}, \gamma_{k}<1, \quad p_{k}<0, \quad q_{k}<0, \quad k=1,2, \ldots, n
$$

are satisfied, then, the solution is unique.

Proof. Now, we consider the corresponding homogeneous problem $[\varphi(y) \equiv \psi(y) \equiv 0]$. Let us estimate the integral $J=\int_{0}^{1} \tau(x) \nu^{+}(x) d x$

We multiply to $\tau(x)$ equation (23) and integrate from 0 to 1 :

$$
\Gamma(\alpha) \int_{0}^{1} \tau(x) \nu^{+}(x) d x=\int_{0}^{1} \tau^{\prime \prime}(x) \tau(x) d x+\int_{0}^{1} \tau(x) \sum_{k=1}^{n} p_{k} I_{0 x}^{-\beta_{k}} \tau(x) d x .
$$

Integrating by parts and using the relations $\tau(0)=\tau(1)=0$, we obtain

$$
J \equiv \int_{0}^{1} \tau(x) \nu^{+}(x) d x=-\int_{0}^{1}\left(\tau^{\prime}(x)\right)^{2} d x+\sum_{k=1}^{n} \frac{p_{k}}{\Gamma\left(\beta_{k}\right)} \int_{0}^{1} \tau(x) d x \int_{0}^{x}(x-t)^{\beta_{k}-1} \tau(t) d t .
$$


By using the formula (see [8], p. 188):

$$
|x-t|^{-\gamma}=\frac{1}{\Gamma(\gamma) \cos \frac{\pi \gamma}{2}} \int_{0}^{\infty} z^{\gamma-1} \cos [z(x-t)] d z, \quad 0<\gamma<1,
$$

after some simplifications, we will obtain (see [3]):

$$
\sum_{k=1}^{n} \frac{1}{\Gamma\left(\beta_{k}\right)} \int_{0}^{1} \tau(x) d x \int_{0}^{x}(x-t)^{\beta_{k}-1} \tau(t) d t \geq 0,
$$

consequently by virtue (24), we will conclude that

$$
\int_{0}^{1} \tau(x) \nu^{+}(x) d x \leq 0
$$

Let us show that $\int_{0}^{1} \tau(x) \nu^{-}(x) d x \geq 0$ for the hyperbolic domain $\Omega^{-}$. For this aim we investigate the integral (see (21)):

$$
A(\eta) \equiv \eta^{\delta} D_{0 \eta}^{1-\delta} \int_{0}^{\eta} \sum_{k=1}^{n} \frac{q_{k} t^{1 / 2}}{\Gamma\left(\gamma_{k}\right)} d t \int_{0}^{t} \frac{\tilde{\tau}(s) d s}{(t-s)^{1-\gamma_{k}}} \times \int_{t}^{\eta} \frac{\eta^{2 \delta}}{(\eta-t)^{\delta} z^{\delta+1 / 2}} F\left(\delta, \delta, 1 ; \frac{t(\eta-z)}{z(\eta-t)}\right) d z .
$$

Entering replacement $\frac{t(\eta-z)}{z(\eta-t)}=\sigma$ and after some simplifications we have:

$$
\begin{gathered}
A(\eta) \equiv \sum_{k=1}^{n} \frac{q_{k}}{\Gamma\left(\gamma_{k}\right) \Gamma(\delta)} \eta^{\delta} \frac{d}{d \eta} \int_{0}^{\eta}(\eta-\mu)^{\delta-1} d \mu \int_{0}^{\mu} t^{1 / 2} d t \int_{0}^{t}(t-s)^{\gamma_{k}-1} \tilde{\tau}(s) d s \times \\
\int_{0}^{1} \frac{\mu^{\delta+1 / 2}[\sigma(\mu-t)+t]^{\delta-3 / 2}}{(\mu-t)^{\delta-1} t^{\delta-1 / 2}} F(\delta, \delta, 1 ; \sigma) d \sigma= \\
\sum_{k=1}^{n} \frac{q_{k}}{\Gamma\left(\gamma_{k}\right) \Gamma(\delta)} \eta^{\delta} \frac{d}{d \eta} \eta^{2+\delta} \int_{0}^{1} \frac{\tilde{\mu}^{\delta+1 / 2} d \tilde{\mu}}{(1-\tilde{\mu})^{1-\delta}} \int_{0}^{\tilde{\mu}} \frac{\tilde{t}^{1-\delta}}{(\tilde{\mu}-\tilde{t})^{\delta-1}} d \tilde{t} \int_{0}^{\tilde{t} \eta} \frac{\tilde{\tau}(s) d s}{(\tilde{t} \eta-s)^{\gamma_{k}-1} \times} \\
\int_{0}^{1}[\sigma(\tilde{\mu}-\tilde{t})+\tilde{t}]^{\delta-3 / 2} F(\delta, \delta, 1 ; \sigma) d \sigma,
\end{gathered}
$$

where

$$
\tilde{\mu}=\mu / \eta, \quad \tilde{t}=t / \eta
$$

Further, taking $\tau(0)=\varphi(0)$ into account and integrating by parts on third integral we will obtain:

$$
\begin{aligned}
& A(\eta) \equiv \varphi(0) \sum_{k=1}^{n} \frac{q_{k}\left(2+\delta+\gamma_{k}\right)}{\Gamma\left(\gamma_{k}+1\right) \Gamma(\delta)} \eta^{1+2 \delta+\gamma_{k}} \int_{0}^{1} \frac{\tilde{\mu}^{\delta+1 / 2} d \tilde{\mu}}{(1-\tilde{\mu})^{1-\delta}} \int_{0}^{\tilde{\mu}} \frac{\tilde{t}^{1+\gamma_{k}-\delta}}{(\tilde{\mu}-\tilde{t})^{\delta-1}} d \tilde{t} \times \int_{0}^{1}[\sigma(\tilde{\mu}-\tilde{t})+\tilde{t}]^{\delta-3 / 2} F(\delta, \delta, 1 ; \sigma) d \sigma+ \\
& \sum_{k=1}^{n} \frac{q_{k}(2+\delta)}{\Gamma\left(\gamma_{k}+1\right) \Gamma(\delta)} \eta^{1+2 \delta} \int_{0}^{1} \frac{\tilde{\mu}^{\delta+1 / 2} d \tilde{\mu}}{(1-\tilde{\mu})^{1-\delta}} \int_{0}^{\tilde{\mu}} \frac{\tilde{t}^{1-\delta}}{(\tilde{\mu}-\tilde{t})^{\delta-1}} d \tilde{t} \int_{0}^{\tilde{t} \eta}(\tilde{t} \eta-s)^{\gamma_{k}} \tilde{\tau}^{\prime}(s) d s \times \int_{0}^{1}[\sigma(\tilde{\mu}-\tilde{t})+\tilde{t}]^{\delta-3 / 2} F(\delta, \delta, 1 ; \sigma) d \sigma+ \\
& \sum_{k=1}^{n} \frac{q_{k}}{\Gamma\left(\gamma_{k}\right) \Gamma(\delta)} \eta^{2+\delta} \int_{0}^{1} \frac{\tilde{\mu}^{\delta+1 / 2} d \tilde{\mu}}{(1-\tilde{\mu})^{1-\delta}} \int_{0}^{\tilde{\mu}} \tilde{t}^{2-\delta}(\tilde{\mu}-\tilde{t})^{1-\delta} d \tilde{t} \int_{0}^{\tilde{t} \eta}(\tilde{t} \eta-s)^{\gamma_{k}-1} \tilde{\tau}^{\prime}(s) d s \times \int_{0}^{1}[\sigma(\tilde{\mu}-\tilde{t})+\tilde{t}]^{\delta-3 / 2} F(\delta, \delta, 1 ; \sigma) d \sigma .
\end{aligned}
$$


By changing the order of integration in the last two terms in (27), we obtain:

$$
\begin{gathered}
A(\eta) \equiv \varphi(0) \sum_{k=1}^{n} \frac{q_{k}\left(2+\delta+\gamma_{k}\right)}{\Gamma\left(\gamma_{k}+1\right) \Gamma(\delta)} \eta^{1+2 \delta+\gamma_{k}} \int_{0}^{1} \frac{\tilde{\mu}^{\delta+1 / 2} d \tilde{\mu}}{(1-\tilde{\mu})^{1-\delta}} \int_{0}^{\tilde{\mu}} \frac{\tilde{t}^{1+\gamma_{k}-\delta} d \tilde{t}}{(\tilde{\mu}-\tilde{t})^{\delta-1}} \times \int_{0}^{1}[\sigma(\tilde{\mu}-\tilde{t})+\tilde{t}]^{\delta-3 / 2} F(\delta, \delta, 1 ; \sigma) d \sigma+ \\
\sum_{k=1}^{n} \frac{q_{k}(2+\delta)}{\Gamma\left(\gamma_{k}+1\right) \Gamma(\delta)} \eta^{1+2 \delta} \int_{0}^{\eta} \tilde{\tau}^{\prime}(s) d s \int_{s / \eta}^{1} \frac{\tilde{\mu}^{\delta+1 / 2} d \tilde{\mu}}{(1-\tilde{\mu})^{1-\delta}} \int_{s / \eta}^{\tilde{\mu}} \frac{\tilde{t}^{1-\delta}(\tilde{t} \eta-s)^{\gamma_{k}} d \tilde{t}}{(\tilde{\mu}-\tilde{t})^{\delta-1}} \times \int_{0}^{1}[\sigma(\tilde{\mu}-\tilde{t})+\tilde{t}]^{\delta-3 / 2} F(\delta, \delta, 1 ; \sigma) d \sigma+ \\
\sum_{k=1}^{n} \frac{q_{k}}{\Gamma\left(\gamma_{k}\right) \Gamma(\delta)} \eta^{2+2 \delta} \int_{0}^{\eta} \tilde{\tau}^{\prime}(s) d s \int_{s / \eta}^{1} \frac{\tilde{\mu}^{\delta+1 / 2} d \tilde{\mu}}{(1-\tilde{\mu})^{1-\delta}} \int_{s / \eta}^{\tilde{\mu}} \tilde{t}^{2-\delta}(\tilde{\mu}-\tilde{t})^{1-\delta}(\tilde{t} \eta-s)^{\gamma_{k}-1} d \tilde{t} \times \\
\int_{0}^{1}[\sigma(\tilde{\mu}-\tilde{t})+\tilde{t}]^{\delta-3 / 2} F(\delta, \delta, 1 ; \sigma) d \sigma .
\end{gathered}
$$

Consequently, using inverse replacements $\mu=\tilde{\mu} \eta$ and $t=\tilde{t} \eta$, we obtain:

$$
\begin{aligned}
& A(\eta) \equiv \varphi(0) \sum_{k=1}^{n} \frac{q_{k}\left(2+\delta+\gamma_{k}\right)}{\Gamma\left(\gamma_{k}+1\right) \Gamma(\delta)} \eta^{\delta-1} \int_{0}^{\eta} \frac{\mu^{\delta+1 / 2} d \mu}{(\eta-\mu)^{1-\delta}} \int_{0}^{\mu} \frac{t^{1+\gamma_{k}-\delta} d t}{(\mu-t)^{\delta-1}} \times \int_{0}^{1}[\sigma(\mu-t)+t]^{\delta-3 / 2} F(\delta, \delta, 1 ; \sigma) d \sigma+ \\
& \sum_{k=1}^{n} \frac{q_{k}(2+\delta)}{\Gamma\left(\gamma_{k}+1\right) \Gamma(\delta)} \eta^{\delta-1} \int_{0}^{\eta} \tilde{\tau}^{\prime}(s) d s \int_{s}^{\eta} \frac{\mu^{\delta+1 / 2} d \mu}{(\eta-\mu)^{1-\delta}} \int_{s}^{\mu} \frac{t^{1-\delta}(t-s)^{\gamma_{k}} d t}{(\mu-t)^{\delta-1}} \times \int_{0}^{1}[\sigma(\mu-t)+t]^{\delta-3 / 2} F(\delta, \delta, 1 ; \sigma) d \sigma+ \\
& \sum_{k=1}^{n} \frac{q_{k}}{\Gamma\left(\gamma_{k}\right) \Gamma(\delta)} \eta^{\delta-1} \int_{0}^{\eta} \tilde{\tau}^{\prime}(s) d s \int_{s}^{\eta} \frac{\mu^{\delta+1 / 2} d \mu}{(\eta-\mu)^{1-\delta}} \int_{s}^{\mu} \frac{t^{2-\delta}(t-s)^{\gamma_{k}-1}}{(\mu-t)^{\delta-1}} d t \times \int_{0}^{1}[\sigma(\mu-t)+t]^{\delta-3 / 2} F(\delta, \delta, 1 ; \sigma) d \sigma .(28)
\end{aligned}
$$

There, the following preliminary assertion holds.

Lemma 1. If a function $\tau(x)$ has a positive maximum (respectively a negative minimum) at the point $x=x_{0} \in$ $(0,1)$, then $\nu^{-}\left(x_{0}\right)>0$ (respectively $\left.\nu^{-}\left(x_{0}\right)<0\right)$ at $q_{k}<0,(k=\overline{1, n})$.

Proof. Let function $\tau(x)$ have a positive maximum at the point $x=x_{0} \in(0,1)$ and $\varphi(y) \equiv 0$, then, from (28), we have:

$$
\begin{gathered}
A\left(x_{0}\right)=\sum_{k=1}^{n} \frac{q_{k}(2+\delta)}{\Gamma\left(\gamma_{k}+1\right) \Gamma(\delta)} x_{0}{ }^{\delta-1} \int_{0}^{x_{0}} \tilde{\tau}^{\prime}(s) d s \int_{s}^{x_{0}} \frac{\mu^{\delta+1 / 2} d \mu}{\left(x_{0}-\mu\right)^{1-\delta}} \int_{s}^{\mu} \frac{t^{1-\delta}(t-s)^{\gamma_{k}}}{(\mu-t)^{\delta-1}} d t \times \\
\int_{0}^{1}[\sigma(\mu-t)+t]^{\delta-3 / 2} F(\delta, \delta, 1 ; \sigma) d \sigma+ \\
\sum_{k=1}^{n} \frac{q_{k}}{\Gamma\left(\gamma_{k}\right) \Gamma(\delta)} x_{0}{ }^{\delta-1} \int_{0}^{x_{0}} \tilde{\tau}^{\prime}(s) d s \int_{s}^{x_{0}} \frac{\mu^{\delta+1 / 2} d \mu}{\left(x_{0}-\mu\right)^{1-\delta}} \int_{s}^{\mu} \frac{t^{2-\delta}(t-s)^{\gamma_{k}-1} d t}{(\mu-t)^{\delta-1}} \times \\
\int_{0}^{1}[\sigma(\mu-t)+t]^{\delta-3 / 2} F(\delta, \delta, 1 ; \sigma) d \sigma .
\end{gathered}
$$

From here, due to $\Gamma\left(\gamma_{k}\right)>0, \Gamma(\delta)>0, q_{k}<0,(k=\overline{1, n}), F(\delta, \delta, 1 ; \sigma)>0$ and

$$
\int_{0}^{x_{0}} \tilde{\tau}^{\prime}(s) d s=\int_{0}^{x_{0}} \lim _{x_{0} \rightarrow s} \frac{\tilde{\tau}\left(x_{0}\right)-\tilde{\tau}(s)}{x_{0}-s} d s>0
$$

we deduce that $A\left(x_{0}\right)<0$. 
In fact, if $\tau(x)$ has a positive maximum at the point $x=x_{0}$ then $D_{0 x_{0}}^{1-2 \delta} \tilde{\tau}\left(x_{0}\right)>0$ (see [19]). Thus, owing to $k_{1}, k_{2}>0$ from (21) at $h(x) \equiv 0$ on the point of positive maximum, we will also deduce:

$$
\tilde{\nu}^{-}\left(x_{0}\right)=\frac{k_{2} \Gamma(1-\delta)}{k_{1} \Gamma(\delta)} x_{0}{ }^{1 / 2} D_{0 x_{0}}^{1-2 \delta} \tilde{\tau}\left(x_{0}\right)-\frac{\Gamma(1-\delta)}{2 k_{1}} A\left(x_{0}\right)>0 .
$$

Similarly, we can prove that on the point of negative minimum $\tilde{\nu}^{-}\left(x_{0}\right)<0$. The lemma is proved.

Hence, based on the Lemma, we will deduce that

$$
\int_{0}^{1} \tau(x) \nu^{-}(x) d x \geq 0
$$

Thus, due to conditions of the Theorem 1 from (29) and (26) we infer that $\tau(x) \equiv 0$. Consequently, from (21), we will obtain $\nu^{-}(x) \equiv 0$.

As a conclusion, based on the solution of the first boundary problem for the Eq.(12), [15], [20] owing to account (14) and (15) we will get $u(x, y) \equiv 0$ in $\bar{\Omega}^{+}$, similarly, based on the solution (20) we obtain $u(x, y) \equiv 0$ in closed domain $\bar{\Omega}^{-}$.

\section{The existence of solution}

Theorem 2. If all conditions of Theorem 1 are satisfied and

$$
\varphi(y), \psi(y) \in C\left(\overline{I_{2}}\right) \cap C^{1}\left(I_{2}\right) ; \quad h(x) \in C^{1}\left(\overline{I_{1}}\right) \cap C^{2}\left(I_{1}\right)
$$

then the solution of the investigating problem is exist.

Proof. Taking (22) into account from Eq.(23) we will obtain

$$
\tau^{\prime \prime}(x)=f(x)
$$

where

$$
f(x)=\lambda \Gamma(\alpha) \nu^{-}(x)-\varphi(0) \sum_{k=1}^{n} \frac{x^{\beta_{k}} p_{k}}{\Gamma\left(\beta_{k}\right)}-\sum_{k=1}^{n} \frac{p_{k}}{\Gamma\left(\beta_{k}\right)} \int_{0}^{x}(x-t)^{\beta_{k}} \tau^{\prime}(t) d t .
$$

Solution of equation (31) together with conditions $\tau(0)=\psi(0), \tau(1)=\varphi(0)$ has the form:

$$
\tau(x)=\int_{0}^{x}(x-t) f(t) d t-x \int_{0}^{1}(1-t) f(t) d t+\varphi(0)(1-x)+x \psi(0),
$$

consequently, we can find:

$$
\tau^{\prime}(x)=\int_{0}^{x} f(t) d t-\int_{0}^{1}(1-t) f(t) d t+\psi(0)-\varphi(0) .
$$

Taking (13) and (28) into account from (21) we will obtain:

$$
\begin{gathered}
\nu^{-}(x)=\tilde{k} \cdot x^{\omega / 2} \int_{0}^{x}\left(x^{\omega}-t^{\omega}\right)^{2 \delta-1} \tau^{\prime}(t) d t- \\
\sum_{k=1}^{n} q_{k}^{1} x^{\frac{\delta-1}{1-2 \delta}} \int_{0}^{x} \tau^{\prime}(s) d s \int_{s}^{x}\left(x^{\omega}-\mu^{\omega}\right)^{\delta-1} \mu^{\frac{6 \delta+1}{2(1-2 \delta)}} d \mu \int_{s}^{\mu} t^{\frac{1+\delta}{1-2 \delta}}\left(\mu^{\omega}-t^{\omega}\right)^{1-\delta} \times \\
\left(t^{\omega}-s^{\omega}\right)^{\gamma_{k}} d t \int_{0}^{1}\left[\sigma\left(\mu^{\omega}-t^{\omega}\right)+t^{\omega}\right]^{\delta-3 / 2} F(\delta, \delta, 1 ; \sigma) d \sigma- \\
\sum_{k=1}^{n} q_{k}^{2} x^{\frac{\delta-1}{1-2 \delta}} \int_{0}^{x} \tau^{\prime}(s) d s \int_{s}^{x}\left(x^{\frac{1}{1-2 \delta}}-\mu^{\frac{1}{1-2 \delta}}\right)^{\delta-1} \mu^{\frac{6 \delta+1}{2(1-2 \delta)}} d \mu \times
\end{gathered}
$$




$$
\begin{gathered}
\int_{s}^{\mu} t^{\frac{2+\delta}{1-2 \delta}}\left(\mu^{\omega}-t^{\omega}\right)^{1-\delta}\left(t^{\omega}-s^{\omega}\right)^{\gamma_{k}-1} d t \int_{0}^{1}\left[\sigma\left(\mu^{\omega}-t^{\omega}\right)+t^{\omega}\right]^{\delta-3 / 2} F(\delta, \delta, 1 ; \sigma) d \sigma- \\
\sum_{k=1}^{n} q_{k}^{3} x^{\frac{\delta-1}{1-2 \delta}} \int_{0}^{x}\left(x^{\omega}-\mu^{\omega}\right)^{\delta-1} \mu^{\frac{6 \delta+1}{2(1-2 \delta)}} d \mu \int_{0}^{\mu} t^{\frac{1+\gamma_{k}+\delta}{1-2 \delta}}\left(\mu^{\omega}-t^{\omega}\right)^{\delta-1} d t \times \\
\int_{0}^{1}\left[\sigma\left(\mu^{\omega}-t^{\omega}\right)+t^{\omega}\right]^{\delta-3 / 2} F(\delta, \delta, 1 ; \sigma) d \sigma+\frac{k_{2} \Gamma(1-\delta)}{k_{1} \Gamma(\delta) \Gamma(2 \delta)} x^{\frac{4 \delta-1}{2(1-2 \delta)}} \varphi(0),
\end{gathered}
$$

where

$$
\begin{gathered}
\omega=\frac{1}{1-2 \delta}, \quad \tilde{k}=\frac{\omega k_{2} \Gamma(1-\delta)}{k_{1} \Gamma(\delta) \Gamma(2 \delta)}, \quad q_{k}^{1}=\frac{(2+\delta) \Gamma(1-\delta) q_{k} \omega^{3}}{2 k_{1} \Gamma\left(\gamma_{k}+1\right) \Gamma(\delta)} \\
q_{k}^{2}=\frac{\Gamma(1-\delta) q_{k} \omega^{3}}{2 k_{1} \Gamma\left(\gamma_{k}\right) \Gamma(\delta)}, \quad q_{k}^{3}=\frac{\Gamma(1-\delta)\left(2+\delta+\gamma_{k}\right) q_{k} \omega^{2}}{2 k_{1} \Gamma(\delta) \Gamma\left(\gamma_{k}+1\right)} \varphi(0) .
\end{gathered}
$$

Further, considering (32) from (33), after some simplifications we will obtain:

$$
\begin{gathered}
\tau^{\prime}(x)=\lambda \Gamma(\alpha) \int_{0}^{x} \nu^{-}(t) d t-\lambda \Gamma(\alpha) \int_{0}^{1}(1-t) \nu^{-}(t) d t-\sum_{k=1}^{n} \frac{p_{k}}{\Gamma\left(\beta_{k}\right)} \int_{0}^{x} d t \int_{0}^{t}(t-s)^{\beta_{k}} \tau^{\prime}(s) d s+ \\
\sum_{k=1}^{n} \frac{p_{k}}{\Gamma\left(\beta_{k}\right)} \int_{0}^{1}(1-t) d t \int_{0}^{t}(t-s)^{\beta_{k}} \tau^{\prime}(s) d s-\varphi(0) \sum_{k=1}^{n} \frac{x^{\beta_{k}+1} p_{k}}{\left(\beta_{k}+1\right) \Gamma\left(\beta_{k}\right)}+ \\
\varphi(0) \sum_{k=1}^{n} \frac{p_{k}}{\left(\beta_{k}+1\right)\left(\beta_{k}+2\right) \Gamma\left(\beta_{k}\right)}+\psi(0)-\varphi(0) .
\end{gathered}
$$

Substituting (34) into (35), we have:

$$
\begin{gathered}
\tau^{\prime}(x)=\tilde{k} \lambda \Gamma(\alpha) \cdot \int_{0}^{x} t^{\frac{3-4 \delta}{2(1-2 \delta)}} d t \int_{0}^{t}\left(t^{\omega}-s^{\omega}\right)^{2 \delta-1} \tau^{\prime}(s) d s-\tilde{k} \lambda \Gamma(\alpha) \cdot \int_{0}^{1}(1-t) t^{\frac{1}{2(1-2 \delta)}} d t \int_{0}^{t}\left(t^{\omega}-s^{\omega}\right)^{2 \delta-1} \tau^{\prime}(s) d s- \\
\lambda \Gamma(\alpha) \sum_{k=1}^{n} q_{k}^{1} \int_{0}^{x} t^{\frac{-\delta}{1-2 \delta}} d t \int_{0}^{t} \tau^{\prime}(s) A(s, t) d s+\lambda \Gamma(\alpha) \sum_{k=1}^{n} q_{k}^{1} \int_{0}^{1}(1-t) t^{\frac{\delta-1}{1-2 \delta}} d t \int_{0}^{t} \tau^{\prime}(s) A(s, t) d s- \\
\lambda \Gamma(\alpha) \sum_{k=1}^{n} q_{k}^{2} \int_{0}^{x} t^{\frac{-\delta}{1-2 \delta}} d t \int_{0}^{t} \tau^{\prime}(s) B(s, t) d s+\lambda \Gamma(\alpha) \sum_{k=1}^{n} q_{k}^{2} \int_{0}^{1}(1-t) t^{\frac{\delta-1}{1-2 \delta}} d t \int_{0}^{t} \tau^{\prime}(s) B(s, t) d s- \\
\sum_{k=1}^{n} \frac{p_{k}}{\Gamma\left(\beta_{k}\right)} \int_{0}^{x} d t \int_{0}^{t}(t-s)^{\beta_{k}} \tau^{\prime}(s) d s+\sum_{k=1}^{n} \frac{p_{k}}{\Gamma\left(\beta_{k}\right)} \int_{0}^{1}(1-t) d t \int_{0}^{\beta_{0}}(t-s)^{\beta_{k}} \tau^{\prime}(s) d s+F(x),
\end{gathered}
$$

where

$$
\begin{gathered}
F(x)=\varphi(0) \sum_{k=1}^{n}\left(\frac{p_{k}}{\left(\beta_{k}+1\right)\left(\beta_{k}+2\right) \Gamma\left(\beta_{k}\right)}-\frac{x^{\beta_{k}+1} p_{k}}{\left(\beta_{k}+1\right) \Gamma\left(\beta_{k}\right)}\right)-\lambda \Gamma(\alpha) \sum_{k=1}^{n} q_{k}^{3} \int_{0}^{x} t^{\frac{\delta-1}{1-2 \delta}} C(t) d t+ \\
\lambda \Gamma(\alpha) \sum_{k=1}^{n} q_{k}^{3} \int_{0}^{1}(1-t) t^{\frac{\delta-1}{1-2 \delta}} C(t) d t-\frac{k_{2} \lambda \Gamma(\alpha) \Gamma(1-\delta)}{k_{1} \Gamma(\delta) \Gamma(2 \delta)} \varphi(0) \int_{0}^{1}(1-t) t^{\frac{4 \delta-1}{2(1-2 \delta)}} d t+ \\
\frac{2(1-2 \delta) k_{2} \lambda \Gamma(\alpha) \Gamma(1-\delta)}{k_{1} \Gamma(\delta) \Gamma(2 \delta)} \varphi(0) x^{\frac{\omega}{2}}-\psi(0)-\varphi(0), \\
A(s, t)=\int_{s}^{t}\left(t^{\omega}-\mu^{\omega}\right)^{\delta-1} \mu^{\frac{6 \delta+1}{2(1-2 \delta)}} d \mu \int_{s}^{\mu} z^{\frac{1+\delta}{1-2 \delta}}\left(\mu^{\omega}-z^{\omega}\right)^{1-\delta} \times
\end{gathered}
$$




$$
\begin{gathered}
\left(z^{\omega}-s^{\omega}\right)^{\gamma_{k}} d z \int_{0}^{1}\left[\sigma\left(\mu^{\omega}-z^{\omega}\right)+z^{\omega}\right]^{\delta-3 / 2} F(\delta, \delta, 1 ; \sigma) d \sigma \\
B(s, t)=\int_{s}^{t}\left(t^{\omega}-\mu^{\omega}\right)^{\delta-1} \mu^{\frac{6 \delta+1}{2(1-2 \delta)}} d \mu \int_{s}^{\mu} z^{\frac{2+\delta}{1-2 \delta}}\left(\mu^{\omega}-z^{\omega}\right)^{1-\delta}\left(z^{\omega}-s^{\omega}\right)^{\gamma_{k}-1} d z \times \\
C(t)=\int_{0}^{t}\left(t^{\omega}-\mu^{\omega}\right)^{\delta-1} \mu^{\frac{6 \delta+1}{2(1-2 \delta)}} d \mu \int_{0}^{\mu} z^{\frac{1+\gamma_{k}+\delta}{1-2 \delta}}\left(\mu^{\omega}-z^{\omega}\right)^{\delta-1} d z \times \\
\int_{0}^{1}\left[\sigma\left(\mu^{\omega}-z^{\omega}\right)+z^{\omega}\right]^{\delta-3 / 2} F(\delta, \delta, 1 ; \sigma) d \sigma,
\end{gathered}
$$

Changing the order of integration in (36), in total, we have integral equation:

$$
\tau^{\prime}(x)=\int_{0}^{1} K(x, s) \tau^{\prime}(s) d s+F(x) .
$$

Here

$$
\begin{gathered}
K(x, s)= \begin{cases}K_{1}(x, s) ; & 0 \leq s \leq x ; \\
K_{2}(x, s) ; & x \leq s \leq 1,\end{cases} \\
K_{1}(x, s)=\lambda \Gamma(\alpha) \int_{s}^{1}(1-t) t^{\frac{\delta-1}{1-2 \delta}}\left[\sum_{k=1}^{n} q_{k}^{1} A(s, t)+\sum_{k=1}^{n} q_{k}^{2} B(s, t)\right] d t+ \\
\lambda \tilde{k} \Gamma(\alpha)\left[\int_{s}^{x} t^{\frac{3-4 \delta}{2-4 \delta}}\left(t^{\omega}-s^{\omega}\right)^{2 \delta-1} d t-\int_{x}^{1}(1-t) t^{\frac{1}{2-4 \delta}}\left(t^{\omega}-s^{\omega}\right)^{2 \delta-1} d t\right]- \\
\lambda \Gamma(\alpha) \int_{s}^{x} t^{\frac{-\delta}{1-2 \delta}}\left[\sum_{k=1}^{n} q_{k}^{1} A(s, t)+\sum_{k=1}^{n} q_{k}^{2} B(s, t)\right] d t+ \\
\sum_{k=1}^{n} \frac{p_{k}}{\Gamma\left(\beta_{k}\right)}\left[\int_{s}^{1}(1-t)(t-s)^{\beta_{k}} d t+\frac{(x-s)^{\beta_{k}+1}}{\beta_{k}+1}\right], \\
K_{2}(x, s)=\lambda \Gamma(\alpha) \int_{s}^{1}(1-t) t^{\frac{\delta-1}{1-2 \delta}}\left[\sum_{k=1}^{n} q_{k}^{1} A(s, t)+\sum_{k=1}^{n} q_{k}^{2} B(s, t)\right] d t- \\
\lambda \tilde{k} \Gamma(\alpha) \int_{s}^{1} \int_{(1-t) t^{\frac{1}{2-4 \delta}}\left(t^{\omega}-s^{\omega}\right)^{2 \delta-1} d t+\sum_{k=1}^{n} \frac{p_{k}}{\Gamma\left(\beta_{k}\right)} \int_{s}(1-t)(t-s)^{\beta_{k}} d t .}
\end{gathered}
$$

In fact, the estimates of functions $A(s, t), B(s, t)$ and $C(t)$ play an important roles for estimating $K(x, s)$. Now, we estimate $|A(s, t)|$. Due to properties of the hypergeometric function (9) from (38), we obtain:

$$
|A(s, t)| \leq\left|\int_{s}^{t}\left(t^{\rho}-\mu^{\rho}\right)^{\delta-1} \mu^{\frac{4 \delta}{2(1-2 \delta)}} d \mu \int_{s}^{\mu} z^{\frac{1+\delta}{1-2 \delta}} \frac{\left(z^{\rho}-s^{\rho}\right)^{\gamma_{k}}}{\left(\mu^{\rho}-z^{\rho}\right)^{\delta}}\right| z^{1 / 2}-\mu^{1 / 2}|d z| .
$$

Introducing the change of variables $z^{\rho}=s^{\rho}+\left(\mu^{\rho}-s^{\rho}\right) z_{1}$ and due to (8), we get

$$
|A(s, t)| \leq\left|t^{1 / 2}-s^{1 / 2}\right| s^{\frac{1}{2-4 \delta}}\left|\int_{s}^{t} \frac{\left(\mu^{\rho}-s^{\rho}\right)^{1-\delta+\gamma_{k}}}{\left(t^{\rho}-\mu^{\rho}\right)^{1-\delta}} \mu^{\frac{4 \delta}{1-2 \delta}} F\left(-\frac{1}{2}, 1+\gamma_{k}, 2+\gamma_{k}-\delta ; \frac{s-\mu}{s}\right) d \mu\right| .
$$


In the sequel, due to

$$
\begin{gathered}
2+\gamma_{k}-\delta-1-\gamma_{k}+\frac{1}{2}=\frac{3}{2}-\delta>0 \\
\left|\frac{s-\mu}{s}\right| \leq 1
\end{gathered}
$$

we have (see (9))

$$
F\left(-\frac{1}{2}, 1+\gamma_{k}, 2+\gamma_{k}-\delta ; \frac{s-\mu}{s}\right) \leq \mathrm{const}
$$

consequently, changing of variables $\mu^{\rho}=s^{\rho}+\left(t^{\rho}-s^{\rho}\right) \mu_{1}$ and by virtue formula (8), after some evaluation finally, we get

$$
|A(s, t)| \leq\left(t^{1 / 2}-s^{1 / 2}\right) s^{\frac{1+4 \delta}{2-4 \delta}}(t-s)^{1+\gamma_{k}} \leq \text { const } .
$$

Similarly, by estimating $B(s, t)$ and $C(t)$ (see (39) and (40)), we can get

$$
|B(s, t)| \leq\left(t^{1 / 2}-s^{1 / 2}\right) s^{\frac{3+4 \delta}{2-4 \delta}}(t-s)^{\gamma_{k}} \leq \text { const, } \quad C(t) \leq \text { const } .
$$

Hence, due to class of given functions (see (30)) and (45), (46) considering (43) and (44) from (37) and (42) respectively we deduce $|K(x, s)| \leq$ const, for all $0 \leq x, s \leq 1,|F(x)| \leq$ const, $0 \leq x \leq 1$. Since kernel $K(x, s)$ is continuous and function in right-side $F(x)$ is continuously differentiable, for the solution of integral equation (41) we can write via resolvent-kernel:

$$
\tau^{\prime}(x)=F(x)-\int_{0}^{1} \Re(x, s) F(s) d s,
$$

where $\Re(x, s)$ is the resolvent-kernel of $K(x, s)$. Unknown functions $\nu^{-}(x)$ we will find from (34). Solution of the Problem I in the domain $\Omega^{+}$we write as follows $[15,20]$ :

$$
\begin{gathered}
u(x, y)=\int_{0}^{y} G_{\xi}(x, y, 0, \eta) \psi(\eta) d \eta-\int_{0}^{y} G_{\xi}(x, y, 1, \eta) \varphi(\eta) d \eta+\int_{0}^{1} G_{0}(x-\xi, y) \tau(\xi) d \xi- \\
\int_{0}^{y} \int_{0}^{1} G(x, y, 0, \eta) \sum_{k=1}^{n} p_{k} I_{0 \xi}^{\beta_{k}} \tau(\xi) d \xi d \eta
\end{gathered}
$$

Here

$$
\begin{gathered}
G_{0}(x-\xi, y)=\frac{1}{\Gamma(1-\alpha)} \int_{0}^{y} \eta^{-\alpha} G(x, y, \xi, \eta) d \eta \\
G(x, y, \xi, \eta)=\frac{(y-\eta)^{\alpha / 2-1}}{2} \sum_{n=-\infty}^{\infty}\left[e_{1, \alpha / 2}^{1, \alpha / 2}\left(-\frac{|x-\xi+2 n|}{(y-\eta)^{\alpha / 2}}\right)-e_{1, \alpha / 2}^{1, \alpha / 2}\left(-\frac{|x+\xi+2 n|}{(y-\eta)^{\alpha / 2}}\right)\right]
\end{gathered}
$$

is the Green's function of the first boundary problem Eq. (12) in the domain $\Omega^{+}$with the Riemann-Liouville fractional differential operator instead of the Caputo ones [20],

$$
e_{1, \delta}^{1, \delta}(z)=\sum_{n=0}^{\infty} \frac{z^{n}}{n ! \Gamma(\delta-\delta n)}
$$

is the Wright type function [18] . Solution of the Problem I in the domain $\Omega^{-}$will be found by the formula (20). Hence, the Theorem 2 is proved.

\section{Acknowledgements}

The authors are grateful to the reviewers for useful suggestions which improve the contents of this paper. 


\section{References}

[1] Nakhushev A. M. The loaded equations and their applications. Nauka, M., 2012.

[2] Abdullaev O. Kh. Non-local Problem for the Loaded Integral-differential Equation in Double-connected Domain. JPDE, 2016, 29(1), P. $1-12$.

[3] Abdullaev O.Kh. About a problem for loaded parabolic-hyperbolictype equations with fractional derivatives. Hindawi Publishing Corporation International Journal of Differential Equations, 2016, Article ID 9815796, 6 p., http: //dx.doi.org/10.1155/2016/9815796.

[4] Kilbas A. A., Srivastava H. M., Trujillo J. J. Theory and Applications of Fractional Differential Equations, in: North-Holland Mathematics Studies, Amsterdam, 2006, (204), Elsevier Science.

[5] Miller K. S., Ross B. An Introduction to the Fractional Calculus and Differential Equations, John Wiley, New York, 1993.

[6] Podlubny I. Fractional Differential Equations, Academic Press, New York, 1999.

[7] Samko S. G., Kilbas A. A., Marichev O. I. Fractional Integral and Derivatives: Theory and Applications. Gordon and Breach, Longhorne, PA, 1993.

[8] Magin R. Fractional calculus in bioengineering, Crit. Rev. Biom. Eng., 2004, 32(1), P. 1-104.

[9] Ortigueira M. Special issue on fractional signal processing and applications, Signal Processing, 2003, 83(11), P. 2285-2480.

[10] Oldham K. B. Fractional differential equations in electrochemistry. Advances in Engineering Software, 2008, P. 12012.

[11] Metzler R, Joseph K. Boundary value problems for fractional diffusion equations, Physics A, 2000, 278, P. $107-125$.

[12] Rivero M. , Trujillo J. J, Velasco M. P., On Deterministic Fractional Models, Edited by D. Baleanu, Ziya B. Guvenc, J.A. Tenreiro Machado in the book New Trends in Nanotechnology and Fractional Calculus Applications, Springer Netherlands, 2010, P. 123-150.

[13] Marichev O. I., Kilbas A. A., Repin O. A. Boundary value problems for partial differential equations with discounting coefficients. Izdat. Samar. Gos. Ekonom. Univ., Samara, 2008 (In Russian).

[14] Repin O. A. Boundary value problems with shift for equations of hyperbolic and mixed type. Saratov Univ., Saratov, 1992 (In Russian).

[15] Karimov E. T., Akhatov J. A boundary problem with integral gluing condition for a parabolic-hyperbolic equation involving the Caputo fractional derivative. EJDE, 2014, 2014, P. 1-6.

[16] Kilbas A. A., Repin O. A. An analog of the Bitsadze-Samarskii problem for a mixed type equation with a fractional derivative. Differential equations, 2003, 39(5), P. 674680.

[17] Kilbas A. A. Repin O. A. An analog of the Tricomi problem for a mixed type equation with a partial fractional derivative. Fractional Calculus and Applied Analysis, 2010, 13(1), P. 6984.

[18] Pskhu A. V. Partial differential equation of fractional order. (Russian). Nauka, Moscow, 2000.

[19] Smirnov M. M. Mixed type equations. M., Nauka, 2000.

[20] Pskhu A. V. Solution of boundary value problems fractional diffusion equation by the Green function method. Differential equation, 2003, 39(10), P. 1509-1513. 